\title{
Biomarker for Asymptomatic Apical Periodontitis in Gingival Crevicular Fluid: aMMP-8
}

\author{
Teodora Karteva $^{1 \oplus}$ Neshka Manchorova-Veleva ${ }^{1}$ \\ ${ }^{1}$ Department of Operative Dentistry and Endodontics, Faculty of \\ Dental Medicine, Medical University - Plovdiv, Plovdiv, Bulgaria
}

\begin{abstract}
Address for correspondence Teodora Georgieva Karteva, PhD, Department of Operative Dentistry and Endodontics, Faculty of Dental Medicine, Medical University - Plovdiv, 15A Vassil Aprilov Boulevard, Plovdiv, 4002, Bulgaria (e-mail: tedy.karteva@gmail.com).
\end{abstract}

\begin{abstract}
Keywords

- asymptomatic apical periodontitis

- gingival crevicular fluid

- aMMP-8

- enzyme linked immunosorbent assay

Objectives Asymptomatic apical periodontitis (AAP) is one of the most widespread chronic inflammatory diseases in the field of dental medicine. Active matrix metalloproteinase (aMMP-8) previously demonstrated diagnostic potential as a biomarker for AAP in gingival crevicular fluid (GCF). The aim of this study was to determine the levels and diagnostic accuracy of aMMP-8 in GCF from teeth with AAP.

Materials and Methods In this cross-sectional study, GCF samples were obtained from teeth with AAP (sample group, $n=31$ ) and their contralateral teeth (control group, $n=31$ ). Clinical and cone beam computed tomography (CBCT) parameters were used for the diagnosis and assessment of AAP and the determination of clinically healthy marginal periodontal tissues. One pool GCF sample per tooth was obtained from the whole crevice's perimeter. aMMP-8 levels were determined by enzyme-linked immunosorbent assay (ELISA).

Statistical Analysis Wilcoxon signed ranks test and Spearman rank correlation coefficient (rs) were used as statistical tools. The significance level was set at $p<.05$.

Results The two groups demonstrated biomarker levels corresponding to a healthy marginal periodontal tissue. aMMP-8 levels were statistically and significantly higher in the samples collected from teeth with AAP. Lesions with greater volume showed correspondingly larger diameters. No statistically significant correlation between aMMP-8 levels and lesions' volume or diameter was discovered.

Conclusion GCF composition is modified by AAP only to a minimal extent. Further research is needed to substantiate the utilization of aMMP- 8 as a potential biomarker for the diagnosis of the disease as well as to explore its relationship with other biomarkers.
\end{abstract}

\section{Introduction}

Asymptomatic apical periodontitis (AAP) is one of the most widespread chronic inflammatory diseases in the field of dental medicine. ${ }^{1}$ AAP is an inflammatory condition of endodontic origin that affects the apical periodontium and the adjacent alveolar bone and cement. It results from the complex interplay between the endodontic biofilm and host immune-inflammatory response. ${ }^{2}$

The egress of bacterial toxins (lipopolysaccharide, lipoteichoic acid, enzymes, and noxious metabolic byproducts) into the periapical tissues activate the innate and adaptive immune system; thus, inducing a periapical inflammatory reaction. ${ }^{3}$ The immunologic response is mediated by numerous cell types that produce a milieu of proinflammatory cytokines, chemokines, and neuropeptides. They induce an alteration in the physiology and metabolism of the periapical tissues. The upregulated cytokine expression results in a shift in the periodontal turnover, ultimately leading to the degradation of extracellular matrix (ECM) components, osteoclastogenesis, and osteoclast activation. ${ }^{4,5}$ ECM degradation of 
periodontal tissues is achieved by intracellular and extracellular pathways. The extracellular pathway entails remodeling by secreted proteases such as matrix metalloproteinases (MMPs). ${ }^{6}$

Members of the MMP family are proteolytic enzymes known to catalyze the hydrolysis of a great variety of biological macromolecules. ${ }^{7}$ In inflammatory periodontal diseases, pathologically excessive activity on their part entails periodontal tissue destruction, which can be monitored by assessing their levels in different oral fluids. ${ }^{8-10}$ On the basis of these biochemical or immunological findings, oral fluids have become a target for extensive research with regard to their diagnostic utilization. ${ }^{11}$ MMPs and their regulators are potential candidates for chair-side or point-of-care (PoC) tests markers, aimed at monitoring periodontal and periimplant diseases., ${ }^{8,12}$ Recent studies demonstrated the diagnostic potential of MMPs found in gingival crevicular fluid (GCF) collected from teeth with AAP. ${ }^{18,19}$ Neutrophil collagenase, also known as matrix metalloproteinase 8 (MMP-8) and it's active form aMMP-8, have been identified as significant collagenolytic enzymes that cause periodontal tissue destruction in periodontitis and their GCF levels are modified by AAP. ${ }^{18,20,21}$

In routine clinical practice, the traditional diagnostic methods are based on clinical-radiographic examination and are designed to evaluate the need for endodontic treatment. These verdict-oriented methods fail to fully delineate the changes observed on a cellular level. The introduction of specific and auspicious biomarkers reflecting AAP does not just provide a new, objective way of diagnosis, but can improve our knowledge of the individual disease dynamics. Therefore, the aim of this cross-sectional study was to establish whether GCF composition can be modified by AAP and assess aMMP- 8 as a potential biomarker for the diagnosis of the disease.

\section{Materials and Methods}

\section{Patient Selection}

The study was approved by the Institutional Review Board of the Medical University of Plovdiv, in accordance with the ethical standards of the Declaration of Helsinki (No. P-3326/20.12.2017). Patients referred to the Department of Operative Dentistry and Endodontics at the university for endodontic treatment with a recommendation for a preoperative cone beam computed tomography (CBCT) were screened for enrollment in the study. A total of 31 patients with a single asymptomatic periapical lesion, intact dentition, clinically healthy marginal periodontium, or gingivitis with no evidence of bone destruction were enrolled in the study. Preoperative CBCT of the teeth with AAP was indicated in all cases due to complex root canal morphology, assessment of nonhealing lesions, or endodontic treatment complications. Limited field of view (FOV) CBCT was taken in accordance with the as low as reasonably achievable (ALARA) principles and the American Association of Endodontists (AAE) and American Academy of Oral and Maxillofacial Radiology (AAOMR) joint position statement on the use of CBCT in endodontics. ${ }^{22,23}$ Exclusion criteria for all subjects included the presence of marginal bone loss, systemic diseases and disorders, smoking, and intake of medication that can influence periodontal tissue turnover in the last 3 months prior to the beginning of the study. Body mass index (BMI) was also taken into consideration as it may affect the GCF levels of MMP-8. ${ }^{24}$ Informed consent was obtained from all the participants.

\section{Radiographic Assessment of the Lesion}

The sample group comprised 31 teeth diagnosed with AAP. The initial assessment of the lesions' characteristics was performed with limited-volume FOV CBCT using dedicated software (Planmeca Romexis Dental Imaging Software; Planmeca OY, Finland). Lesion size was determined with the help of its diameter and volume. The diameter was deemed to be the most substantial cross-section perpendicular to the root surface in the apical third of the root. The volumetric measurements were calculated utilizing two different techniques-manual outlining of the lesion borders with a consequent automatic reconstruction of the area and automatic threshold volume generation. Each periapical lesion was located and assessed in the three planes (axial plane, sagittal plane, and coronal plane). The measure ellipsoid tool was used to select the area of the lesion. Automatic volume threshold generation at 300 to 350 Hounsfield units was performed ( - Fig. 1). The threshold value was the most optimal value that yielded complete outlining of the lesion. The software consequently automatically calculated the lesion volume. The second method of volume determination comprised manual outlining of the lesion in each slice. Automatic reconstruction and calculation followed ( - Fig. 2).

\section{Clinical Phase}

All patients underwent initial periodontal therapy-periodontal screening and recording and calculus removal were performed, followed by oral hygiene instruction and motivation. All patient data was recorded in a medical chart that included medical and dental history, smoking habits, medication intake, and periodontal clinical parameters. Periodontal probing depth (PPD), bleeding on probing (BOP), and bleeding index (BI) were measured using a calibrated periodontal probe (UNC-15; Hu-Friedy, Chicago, IL, USA) in six sites of

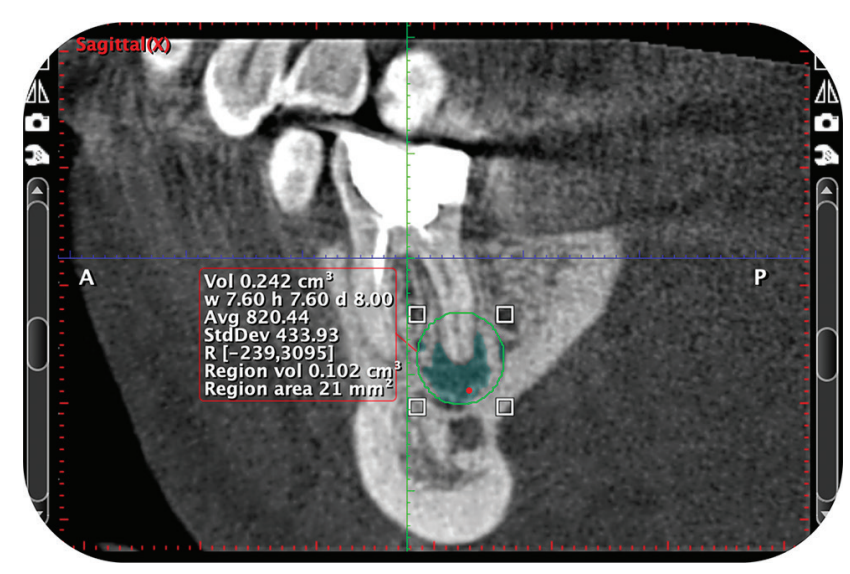

Fig. 1 Lesion volume determination: automatic threshold volume generation. 


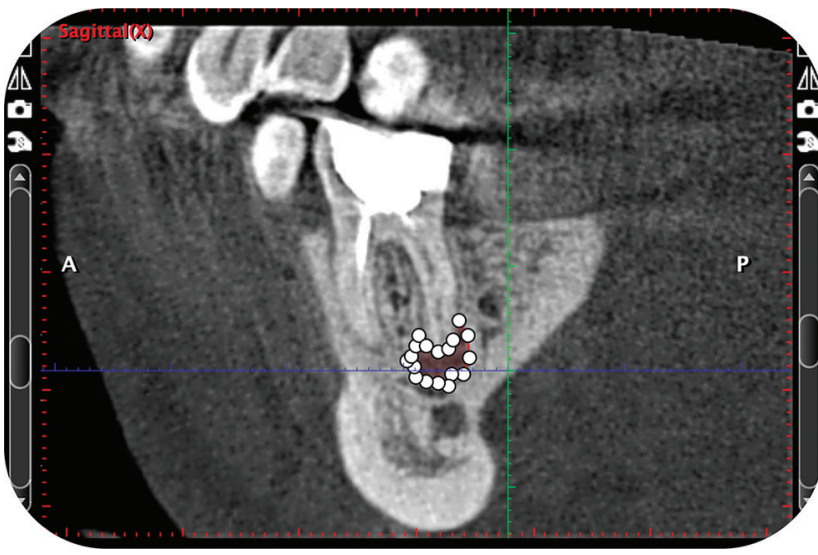

Fig. 2 Lesion volume determination: manual outlining of the lesion borders with a consequent automatic reconstruction.

each tooth diagnosed with AAP (the sample group, $n=31$ ) and its periodontally healthy contralateral tooth (the control group, $n=31$ ). Pulp vitality testing was performed on the contralateral teeth to confirm intact pulp and periapical tissues. Patients were recalled in 2 weeks for reassessment and evaluation of the response of the initial therapy. No BOP and a probing depth of $\leq 3 \mathrm{~mm}$ on the sites to be sampled were a prerequisite for proceeding with the sampling procedures.

\section{Sample Collection}

Customized sample taking kits containing six GCF-stripes, one tube, and one patient form were provided by Dentognostics GmbH (Jena, Germany). One Pool GCF sample per tooth was obtained intracrevicularly from the whole crevice's perimeter (mesiovestibular, vestibular, distovestibular, distolingual, lingual, and mesiolingual) of the teeth in the sample and control group. The teeth were isolated with cotton rolls and the sulcus was dried. Each paper strip was gently inserted in the gingival sulcus for 30 seconds ( - Fig. 3). Strips contaminated with blood or saliva were discarded and the samples were sent to the manufacturer. aMMP-8 levels were determined by laboratory-based enzyme-linked immunosorbent assay (ELISA) (DentoELISA; Dentognostics GmbH, Jena, Germany). Data was typed into a spreadsheet and SPSS software (version 18.0) (SPSS Inc.; Chicago, Illinois, United States) was used to perform the analysis. Wilcoxon signed ranks test and Spearman rank correlation coefficient (rs) were used as statistical tools. The significance level was set at $p<.05$.

\section{Results}

Ten female and 21 male patients were assessed in the study with a mean age of 26.4 (range 21-33). The age, gender, BMI, and the number of teeth of the study subjects are shown in - Table 1. The clinical periodontal parameters are shown in - Table 2. The levels of aMMP-8 were assessed in pool GCF samples collected from 31 diseased teeth and 31 control contralateral teeth. All teeth in the sample group were multirooted, 10 maxillary, and 21 mandibular. Of those, 18 were retreatment cases. Both the sample and the control group demonstrated biomarker levels corresponding to a

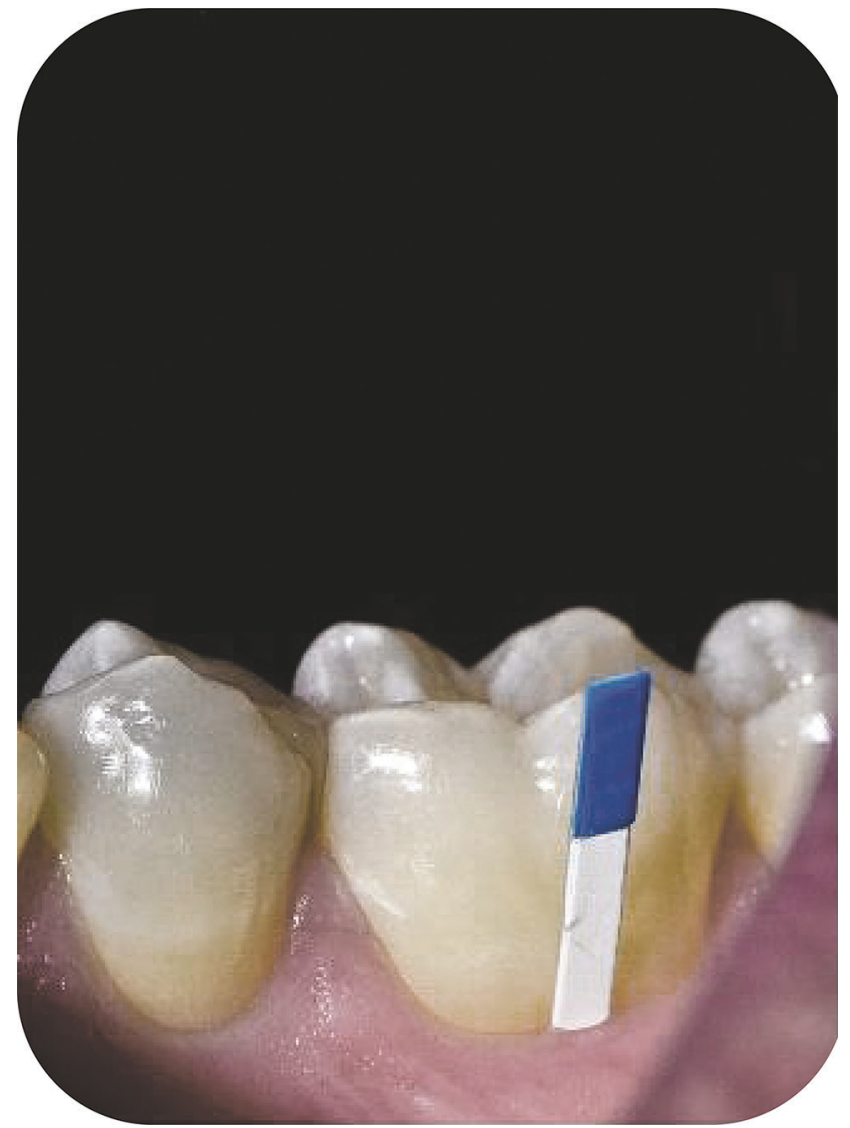

Fig. 3 GCF sample collection. GCF, gingival crevicular fluid.

healthy periodontal tissue ( - Fig. 4). aMMP-8 levels were not affected by age, gender, BMI, tooth location, or previous endodontic treatment. The mean levels of aMMP-8 in the sample group were $6.65 \mathrm{ng} / \mathrm{mL}$ and $3.77 \mathrm{ng} / \mathrm{mL}$ in the control group (-Table 3). A statistically significant difference between the two groups was established as the levels of aMMP-8 were higher in the GCF, collected from teeth with AAP $(Z=-3.45$, $p<.001)$. Lesions with greater volume showed correspondingly larger diameters $\left(r_{s}=0.705, p=.000\right)$. A nonsignificant fair positive correlation was discovered between aMMP-8 levels and lesion diameter $\left(r_{s}=0.338, p=.063\right)$. No significant correlation between aMMP-8 levels and lesion volumes was established.

\section{Discussion}

GCF is a plasmatic extravasate collected noninvasively at the gingival margin or within the gingival crevice. It has a highdiagnostic potential as a source of biomarkers for marginal periodontal disease. ${ }^{19,20,25-27}$ The biochemical analysis of GCF can be directed at components derived from the host: cytokines and other inflammatory mediators. ${ }^{28}$ Utilizing GCF biomarkers for the assessment of marginal periodontal diseases represents a widespread concept. The biomarkers directly account for the disease's inflammatory profile as a complement to the existing clinical-radiographic assessment. ${ }^{18}$

According to Baeza et al, GCF can also reflect the condition of the apical periodontium, although the extent to which AAP 
Table 1 Age, gender, body mass index, and number of teeth of the study subjects

\begin{tabular}{|l|l|l|l|l|l|l|l|}
\hline \multicolumn{2}{|c|}{ Gender } & \multicolumn{2}{c|}{ Age } & \multicolumn{2}{c|}{ BMI } & \multicolumn{3}{c|}{ Number of teeth } \\
\hline Male & Female & Mean & $95 \% \mathrm{Cl}$ & Mean & $95 \% \mathrm{Cl}$ & $\mathrm{Mean}$ & $95 \% \mathrm{Cl}$ \\
\hline 21 & 10 & 26.4 & $25.0,27.8$ & 25.39 & $24.39,26.39$ & 29 & 28,30 \\
\hline
\end{tabular}

Abbreviations: BMI, body mass index; $\mathrm{Cl}$, confidence interval.

Table 2 Clinical parameters of 372 tooth sites from 61 teeth

\begin{tabular}{|l|l|l|l|l|l|l|}
\hline \multirow{2}{*}{ Group } & \multirow{2}{*}{ Sites } & \multicolumn{2}{|c|}{ PD } & \multicolumn{2}{c|}{ BI } & BOP \\
\cline { 2 - 7 } & & Mean & $95 \%$ Cl & Mean & $95 \%$ & CI \\
\hline Sample & 186 & 2.03 & $1.93,2.13$ & 1.96 & $1.83,2.09$ & 32.3 \\
\hline Control & 186 & 2.09 & $1.99,2.19$ & 1.75 & $1.62,1.88$ & 23.1 \\
\hline Total & 372 & 2.06 & $1.99,2.13$ & 1.85 & $1.76,1.95$ & 27.7 \\
\hline
\end{tabular}

Abbreviations: BI, bleeding index; BOP, bleeding on probing; $\mathrm{Cl}$, confidence interval; PPD, periodontal probing depth.

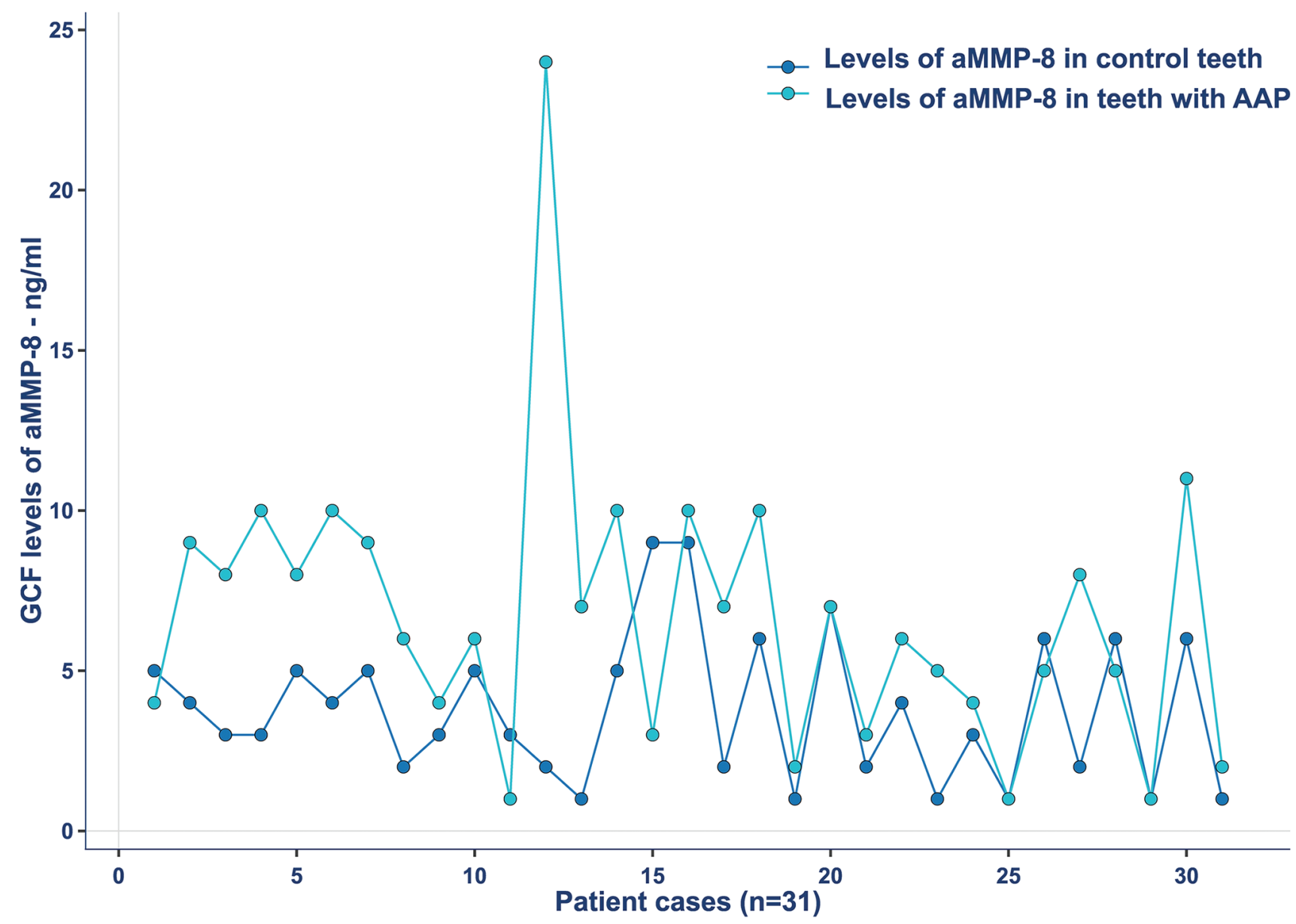

Fig. 4 Individual levels of aMMP-8 in control teeth and teeth with AAP. AAP, asymptomatic apical periodontitis, aMMP, active matrix metalloproteinase.

Table 3 Levels of aMMP-8 in GCF pool samples from 31 patents $(\mathrm{ng} / \mathrm{mL})$

\begin{tabular}{|l|l|l|l|l|l|l|}
\hline Group & N & Mean & $95 \%$ Cl & Min & Max & Med \\
\hline Sample & 31 & 6.65 & $5.02,8.27$ & 1 & 24 & 6 \\
\hline Control & 31 & 3.77 & $2.93,4.62$ & 1 & 9 & 3 \\
\hline
\end{tabular}

Abbreviations: aMMP, active matrix metalloproteinase; $\mathrm{Cl}$, confidence interval; GCF, gingival crevicular fluid. 
can modify GCF remains mostly unexplored..$^{18}$ The present study investigated aMMP-8 as a potential biomarker for the disease. Increased levels of aMMP-8 in oral fluids are associated with clinical periodontal parameters (PPD and BOP) and decrease after successful periodontal treatment. ${ }^{29,30}$ This presented a challenge, as any gingival inflammation would affect the detection of AAP biomarkers in GCF. We therefore suggested limiting the applicability of the method to teeth with a clinically healthy marginal periodontium in order to reduce the confounding effect of gingival inflammation. Comparing the results to a contralateral tooth with an intact apical periodontium will also provide a baseline for interpreting the results.

Previous studies validated a $20 \mathrm{ng} / \mathrm{mL}$ clinical threshold of aMMP-8 GCF levels, which indicates marginal periodontal degeneration. ${ }^{31-35}$ This serves as the detection limit for the commercially available chair-side and PoC lateral flow immunotests for the detection of aMMP-8 in oral fluids (i.e., PerioSafe and ImplantSafe). Baeza et al suggests a cutoff point at $13 \mathrm{ng} / \mathrm{mL}$ in laboratory-based lateral flow immunoassay of aMMP-8 for chronic periodontitis. ${ }^{18} \mathrm{We}$ observed lower aMMP-8 levels in the majority of samples from the sample and control groups alike. As the sampled sites did not show any signs gingival inflammation at the time of sample collection, the results are in accordance with previously reported levels for healthy marginal periodontium. The low levels of aMMP-8 could also be attributed to the small volume of GCF produced in a clinically healthy marginal crevice. Nevertheless, aMMP-8 levels were higher in the GCF collected from teeth with AAP in comparison with the contralateral sample $(p=0.001)$. Therefore, the current qualitative chair-side and PoC GCF tests could not be utilized for the screening of AAP due to their high-detection threshold. Future studies should focus on laboratory-based methods as they have higher sensitivity.

The extent of bone destruction in terms of lesion diameter and volume was assessed by CBCT. In developing chronic periodontitis and peri-implantitis the collagen degradation can be detected by aMMP-8 tests prior to any clinical and radiographic sighs. ${ }^{36}$ Given the high sensitivity of the method, we supposed larger apical lesions would be associated with higher aMMP-8 levels, even though no clinical symptoms for disease activity were present in our patients. However, only a fair positive correlation was discovered between aMMP-8 levels and lesion diameter, and the results did not reach statistical significance. One possible explanation for this finding could be the dynamics of AAP that is characterized with little lesion expansion and minimal bone resorption. ${ }^{37}$ It is possible that GCF biomarkers will be increased in the active phase of the disease when the lesion is initially formed. Accordingly, the proposed method could be utilized in the short-term follow-up of endodontically treated teeth with no periodontal involvement to confirm the success of the intervention and monitor the development of periapical lesions.

Even though the observed impact of AAP on GCF composition seems to be minimal, our results suggest aMMP-8 in teeth with AAP reaches the gingival crevice. Beaza et al suggested two possible mechanisms of the observed phenomenon: diffusional and circulatory. ${ }^{18}$ According to the former, the molecules might leak through the periodontal ligament. In the circulatory mechanism, the molecules enter the periapical blood vessels and reach the subepithelial plexus via the anastomoses between the vessels of the periodontal ligament and the supraperiosteal blood vessels. These findings could be significant as biomarkers found in local circulation can impact systemic circulation as well. According to recent studies, the molecules can reach the general circulation, eliciting a low-grade inflammatory response..$^{25,38,39}$ The results of the present study can be explained by the circulatory mechanism proposed by Baeza et al, which, in turn, can serve as an underlying mechanisms of the systemic response to periapical disease. AAP can, therefore, be a potential risk factor for inflammatory systemic diseases such as cardiovascular diseases or depression. ${ }^{40} \mathrm{As}$ insufficient treatment motivation is commonly observed as a result of the equivocal clinical presentation of AAP, mounting evidence of the systemic impact of the disease could improve treatment compliance.

\section{Conclusion}

The results suggested that GCF composition is modified by AAP only to a minimal extent but signifies a possible underlying mechanism of how the release of proinflammatory mediators contributes to low-grade systemic inflammation. As demonstrated in the present study, the levels of activation of MMP-8 in GCF do not increase to pathologically elevated levels in teeth with AAP. Further research is needed to substantiate the use of aMMP-8 as a potential biomarker for the diagnosis of the disease as well as explore its relationship with other biomarkers. The detection of GCF biomarkers reflecting the periapical status can aid clinical diagnosis and shortterm follow-up. In line with molecular diagnostic trends, this approach can augment the current evaluation methods, promote the successful personalization of AAP treatment, and optimize the long-term prognosis of the disease.

\section{Funding}

This study received a grant from the Medical University Plovdiv, under Grant Contract N DPDP-13/2018.

\section{Conflict of Interest}

None declared.

\section{References}

1 Persoon IF, Özok AR. Definitions and epidemiology of endodontic infections. Curr Oral Health Rep 2017;4(4):278-285

2 Graves DT, Oates T, Garlet GP. Review of osteoimmunology and the host response in endodontic and periodontal lesions. J Oral Microbiol 2011;3(1):5304

3 Dwyer TG, Torabinejad M. Radiographic and histologic evaluation of the effect of endotoxin on the periapical tissues of the cat. J Endod 1981;7(1):31-35

4 Bartold PM, Cantley MD, Haynes DR. Mechanisms and control of pathologic bone loss in periodontitis. Periodontol 2000 2010;53(1):55-69 
5 Liu YC, Lerner UH, Teng YT. Cytokine responses against periodontal infection: protective and destructive roles. Periodontol 2000 2010;52(1):163-206

6 Sagi I, Matrix Metalloproteinase Biology. Hoboken, NJ: Wiley Blackwell; 2015

7 Rawlings ND, Waller M, Barrett AJ, Bateman A. MEROPS: the database of proteolytic enzymes, their substrates and inhibitors. Nucleic Acids Res 2014;42(Database issue) (D1): D503-D509

8 Sorsa T, Tjäderhane L, Salo T. Matrix metalloproteinases (MMPs) in oral diseases. Oral Dis 2004;10(6):311-318

9 Sorsa T, Tjäderhane L, Konttinen YT, et al. Matrix metalloproteinases: contribution to pathogenesis, diagnosis and treatment of periodontal inflammation. Ann Med 2006;38(5):306-321

10 Salazar MG, Jehmlich N, Murr A, et al. Identification of periodontitis associated changes in the proteome of whole human saliva by mass spectrometric analysis. J Clin Periodontol 2013;40(9):825-832

11 Gupta N, Gupta ND, Gupta A, Goyal L, Garg S. The influence of type 2 diabetes mellitus on salivary matrix metalloproteinase-8 levels and periodontal parameters: a study in an Indian population. Eur J Dent 2015;9(3):319-323

12 Giannobile WV. Host-response therapeutics for periodontal diseases. J Periodontol 2008;79(8 (Suppl):1592-1600

13 Herrera D, Matesanz P, Bascones-Martínez A, Sanz M. Local and systemic antimicrobial therapy in periodontics. J Evid Based Dent Pract 2012;12(3 (Suppl):50-60

14 Khiste SV, Ranganath V, Nichani AS, Rajani V. Critical analysis of biomarkers in the current periodontal practice. J Indian Soc Periodontol 2011;15(2):104-110

15 Miller CS, Foley JD, Bailey AL, et al. Current developments in salivary diagnostics. Biomarkers Med 2010;4(1):171-189

16 Sorsa T, Mäntylä P, Tervahartiala T, Pussinen PJ, Gamonal $\mathrm{J}$, Hernandez M. MMP activation in diagnostics of periodontitis and systemic inflammation. J Clin Periodontol 2011;38(9):817-819

17 Sorsa T, Tervahartiala T, Leppilahti J, et al. Collagenase-2 (MMP-8) as a point-of-care biomarker in periodontitis and cardiovascular diseases. Therapeutic response to non-antimicrobial properties of tetracyclines. Pharmacol Res 2011;63(2):108-113

18 Baeza M, Garrido M, Hernández-Ríos P, et al. Diagnostic accuracy for apical and chronic periodontitis biomarkers in gingival crevicular fluid: an exploratory study. J Clin Periodontol 2016;43(1):34-45

19 Dezerega A, Madrid S, Mundi V, et al. Pro-oxidant status and matrix metalloproteinases in apical lesions and gingival crevicular fluid as potential biomarkers for asymptomatic apical periodontitis and endodontic treatment response. J Inflamm (Lond) 2012;9(1):8

20 Belmar MJ, Pabst C, Martínez B, Hernández M. Gelatinolytic activity in gingival crevicular fluid from teeth with periapical lesions. Oral Surg Oral Med Oral Pathol Oral Radiol Endod 2008;105(6):801-806

21 Kinane DF, Stathopoulou PG, Papapanou PN. Periodontal diseases. Nat Rev Dis Primers 2017;3(1):17038

22 Fayad MI, Nair M, Levin MD, et al. AAE and AAOMR joint position statement. J Endod 2011;37(2):274-277

23 Fayad MI, Nair M, Levin MD, et al; Special Committee to Revise the Joint AAE/AAOMR Position Statement on Use of CBCT in Endodontics. AAE and AAOMR Joint Position Statement: Use of Cone Beam Computed Tomography in Endodontics 2015 Update. Oral Surg Oral Med Oral Pathol Oral Radiol 2015;120(4):508-512
24 Park HS, Nam HS, Seo HS, Hwang SJ. Change of periodontal inflammatory indicators through a 4-week weight control intervention including caloric restriction and exercise training in young Koreans: a pilot study. BMC Oral Health 2015;15(1):109

25 Burgener B, Ford AR, Situ H, et al. Biologic markers for odontogenic periradicular periodontitis. J Endod 2010;36(8): 1307-1310

26 Leppilahti JM, Hernández-Ríos PA, Gamonal JA, et al. Matrix metalloproteinases and myeloperoxidase in gingival crevicular fluid provide site-specific diagnostic value for chronic periodontitis. J Clin Periodontol 2014;41(4):348-356

27 Buduneli N, Kinane DF. Host-derived diagnostic markers related to soft tissue destruction and bone degradation in periodontitis. J Clin Periodontol 2011;38(Suppl 11):85-105

28 Arias-Bujanda N, Regueira-Iglesias A, Alonso-Sampedro M, et al. Cytokine thresholds in gingival crevicular fluid with potential diagnosis of chronic periodontitis differentiating by smoking status. Sci Rep 2018;8(1):18003

29 Mauramo M, Ramseier AM, Mauramo E, et al. Associations of oral fluid MMP-8 with periodontitis in Swiss adult subjects. Oral Dis 2018;24(3):449-455

30 Sorsa T, Gursoy UK, Nwhator S, et al. Analysis of matrix metalloproteinases, especially MMP-8, in gingival creviclular fluid, mouthrinse and saliva for monitoring periodontal diseases. Periodontol 2000 2016;70(1):142-163

31 Heikkinen AM, Nwhator SO, Rathnayake N, Mäntylä P, Vatanen P, Sorsa T. Pilot study on oral health status as assessed by an active matrix metalloproteinase- 8 chairside mouthrinse test in adolescents. J Periodontol 2016;87(1):36-40

32 Heikkinen AM, Raivisto T, Kettunen K, et al. Pilot study on the genetic background of an active matrix metalloproteinase- 8 test in Finnish adolescents. J Periodontol 2017;88(5):464-472

33 Alassiri S, Parnanen P, Rathnayake N, et al. The ability of quantitative, specific, and sensitive point-of-care/chair-side oral fluid immunotests for ammp-8 to detect periodontal and peri-implant diseases. Dis Markers 2018;2018:1306396

34 Leppilahti JM, Harjunmaa U, Järnstedt J, et al. Diagnosis of newly delivered mothers for periodontitis with a novel oralrinse ammp-8 point-of-care test in a rural Malawian population. Diagnostics (Basel) 2018;8(3):67

35 Lorenz K, Keller T, Noack B, Freitag A, Netuschil L, Hoffmann T. Evaluation of a novel point-of-care test for active matrix metalloproteinase-8: agreement between qualitative and quantitative measurements and relation to periodontal inflammation. J Periodontal Res 2017;52(2):277-284

36 Sorsa T, Gieselmann D, Arweiler NB, Hernández M. A quantitative point-of-care test for periodontal and dental peri-implant diseases. Nat Rev Dis Primers 2017;3(1):17069

37 Stashenko P, Yu SM, Wang CY. Kinetics of immune cell and bone resorptive responses to endodontic infections. J Endod 1992;18(9):422-426

38 Gomes MS, Blattner TC, Sant'Ana Filho M, et al. Can apical periodontitis modify systemic levels of inflammatory markers? A systematic review and meta-analysis. J Endod 2013;39(10):1205-1217

39 Georgiou AC, Crielaard W, Armenis I. de Vries R, van der Waal SV. Apical periodontitis is associated with elevated concentrations of inflammatory mediators in peripheral blood: a systematic review and meta-analysis. J Endod 2019;45(11):1279-1295

40 Gomes C, Martinho FC, Barbosa DS, et al. Increased root canal endotoxin levels are associated with chronic apical periodontitis, increased oxidative and nitrosative stress, major depression, severity of depression, and a lowered quality of life. Mol Neurobiol 2018;55(4):2814-2827 